\title{
Atomic structure via highly charged ions and their exact quantum states
}

\author{
G. Friesecke ${ }^{1,2}$ and B. D. Goddard ${ }^{2}$ \\ ${ }^{1}$ Center for Mathematics, TU Munich, Germany \\ ${ }^{2}$ Mathematics Institute, University of Warwick, Coventry CV47AL, United Kingdom
}

(Received 18 September 2009; published 25 March 2010)

\begin{abstract}
For highly charged ions with 3 to 10 electrons, we derive explicit, closed-form quantum states which become exact in the high charge limit. When combined with suitably renormalized experimental data across isoelectronic sequences, these quantum states provide a widely applicable route to predicting electronic configurations and term values for neutral atom energy levels. Moreover, our findings allow the prediction of missing levels, suggest that certain current term assignments for five-electron ions are incorrect, and provide insight into the rare failure of Hund's rules in excited states.
\end{abstract}

DOI: 10.1103/PhysRevA.81.032516

PACS number(s): 31.15.ac, 31.15.ae, 32.30.-r

\section{INTRODUCTION}

It has long been recognized by experimental spectroscopists [1,2] and quantum theorists [3-7] that highly charged ions provide an attractive setting for the detailed understanding of electronic structure and spectral properties of many-electron systems. Highly charged ions are also of direct interest in many contexts, e.g., strong field experiments in quantum electrodynamics [8], plasma physics [9], and the investigation of parity nonconservation [10].

Here we report, for highly charged ions with 3 to 10 electrons, explicit, closed-form quantum states which become exact in the high charge limit. The ground states are surprisingly similar to the semiempirical hydrogen orbital configurations going back to Bohr, Hund, Pauli, and Slater [11-13]. Our exact quantum states provide insight into the fundamental mechanisms by which atomic structure emerges from quantum mechanics. In particular, they yield a method for term and configuration assignment for neutral atoms, by using suitably renormalized experimental levels of the corresponding isoelectronic sequence, such as $\mathrm{Li}, \mathrm{Be}^{+}, \mathrm{B}^{2+}$, $\mathrm{C}^{3+}, \ldots$ to interpolate to high charge ions. Also, our findings suggest that certain current term assignments for five-electron ions are incorrect, allow the prediction of missing levels, and offer theoretical insight into Hund's rules and their occasional failure.

\section{THEORETICAL BACKGROUND}

Starting point of all theoretical insight into atomic energy levels and states is the time-independent Schrödinger equation

$$
H \Psi=E \Psi
$$

where $H$ is the Hamiltonian of the system, $E$ is the energy, and $\Psi$ is the wave function of the electrons.

For the lighter atoms, relativistic effects can be neglected and, for nuclear charge $Z$ and $N$ electrons and in atomic units, the (Born-Oppenheimer) Hamiltonian is

$$
H=\sum_{i=1}^{N}\left(-\frac{1}{2} \nabla_{i}^{2}-\frac{Z}{r_{i}}\right)+\sum_{1 \leqslant i<j \leqslant N} \frac{1}{r_{i j}} .
$$

Here the $r_{i}$ and $r_{i j}$ are the electron-nucleus and electronelectron distances, respectively, and $\nabla_{i}$ is the gradient with respect to the position coordinates $x_{i}$ of the $i$ th electron. The wave function $\Psi$ depends on the position coordinates and spins of all the electrons, and must be antisymmetric with respect to simultaneous exchange of the positions and spins of any two electrons, by the Pauli principle.

\section{QUANTUM STATES}

Our results concern isoelectronic sequences. These are defined by holding the number $N$ of electrons fixed and increasing the nuclear charge $Z$, as in the lithium sequence $\mathrm{Li}, \mathrm{Be}^{+}, \mathrm{B}^{2+}, \mathrm{C}^{3+}, \ldots$ We find that in the large $Z$ limit, the low-lying quantum states can be determined explicitly, in closed form. The ground states for 1 to 10 electrons are shown in Fig. 1 and Table I. For excited states see Table II. The status of these quantum states is given by the following mathematical theorem: the difference between the true solutions to the Schrödinger equation (1) with Hamiltonian (2), and the simple wave functions given in the tables, tends in a least-squares sense to zero along each isoelectronic sequence.

The derivation of these results is outlined in an Appendix. The full details are documented elsewhere [14]. In an interesting previous study [4], such asymptotic wave functions are derived, but those given for $\mathrm{B}, \mathrm{Be}$, and $\mathrm{C}$ were incorrect, being the standard hydrogen orbital configurations.

We note that the best available neutral atom or small molecule wave functions delivered by computational methods [15] consist of a superposition of millions [16] or even billions [17] of different (method dependent) configurations, with the exact functions requiring an infinite superposition [18]. The simplicity of the wave functions derived here from (1) and (2) for ions is then remarkable and lends theoretical support to the continuing use of simplified wave functions in the atomic spectra database [20] and their ongoing use as a source of physical and chemical intuition and a starting point for designing reduced models of complex systems [21-24].

We now compare the ground states in Fig. 1 to the semiempirical hydrogen orbital configurations developed by Bohr, Hund, and Slater [11-13] to explain the periodic table. Recall the underlying rules: (a) Each electron in an atom occupies a hydrogenic orbital. ${ }^{1}$ (b) The orbitals in each

\footnotetext{
${ }^{1}$ In fact, in Bohr's and Hund's original works [11,12] the electrons were supposed to occupy hydrogenic Bohr orbits.
} 


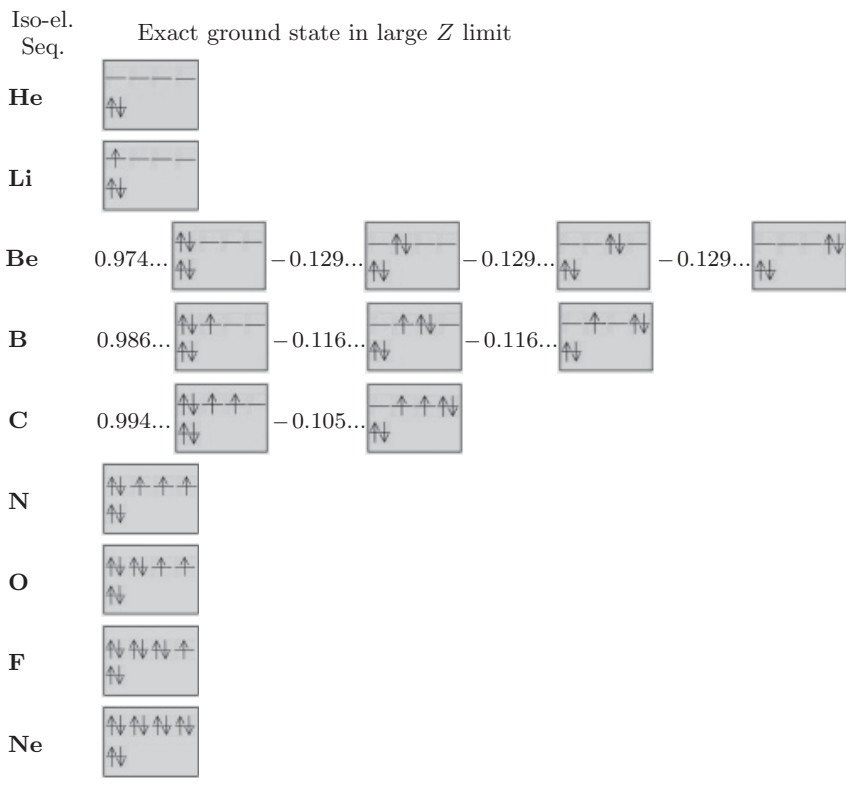

FIG. 1. Diagrammatic representation of the ground states of the Schrödinger equations (1) and (2) in the large $Z$ limit. Arrows indicate spin-up and spin-down electrons occupying the four lowest hydrogen levels ( $1 s$, bottom; $2 s$, top left; $2 p_{i}, i=1,2,3$, top right). Note the close similarity with the semiempirical hydrogen orbital configurations. For more details see Table I.

hydrogen energy level, or shell, form sub-shells which are occupied in the order $1 s, 2 s, 2 p, 3 s, 3 p, 4 s, 3 d, \ldots$ (c) Hund's rule: Within any partially filled subshell, the electrons adopt a configuration with the greatest possible number of aligned spins. Thus, in, say, the carbon sequence, the six electrons would occupy the orbitals $1 s \overline{1 s} 2 s \overline{2 s} 2 p_{1} 2 p_{2}$ [the alternative choices $\overline{2 p_{1}}$ or $\overline{2 p_{2}}$ for the last orbital are consistent with (b) but not (c)].

There is a long history of explaining this beautiful heuristic picture in terms of numerical solutions of Hartree- and HartreeFock models [25]; here, for highly charged ions it is seen to emerge directly from the fundamental laws of quantum mechanics. For 7 of 10 elements, the high-charge limit of the

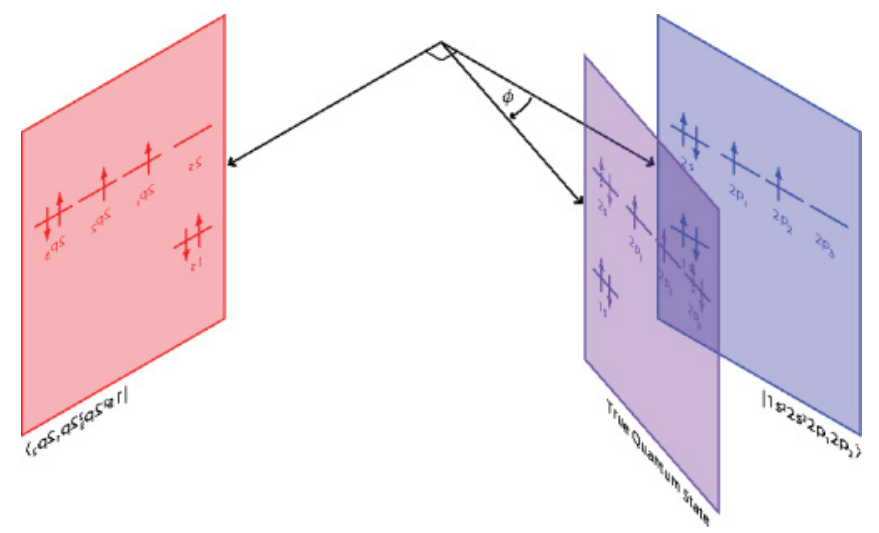

FIG. 2. (Color) Hilbert space visualization of the large- $Z$ six-electron ion ground state. Note that it can be written in the form $\cos \phi\left|1 s^{2} 2 s^{2} 2 p_{1} 2 p_{2}\right\rangle-\sin \phi\left|1 s^{2} 2 p_{1} 2 p_{2} 2 p_{3}^{2}\right\rangle$, with the nonobvious angle $\phi=\arctan [(\sqrt{221876564389}-460642) / 98415] \approx 6^{\circ}$.

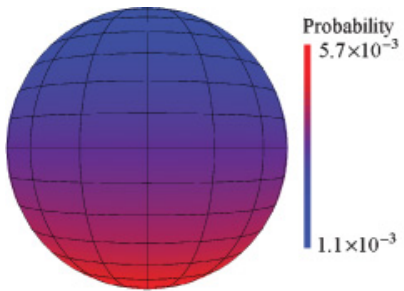

(a)

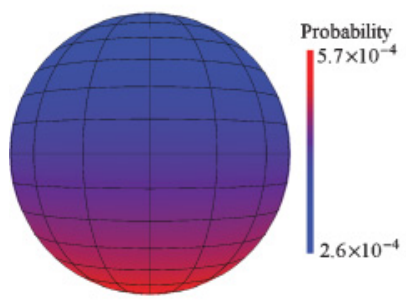

(c)

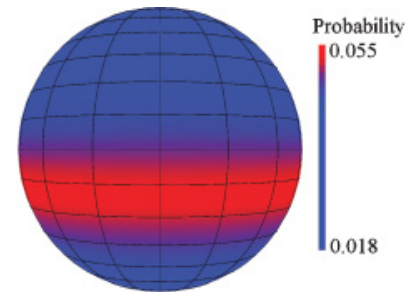

(b)

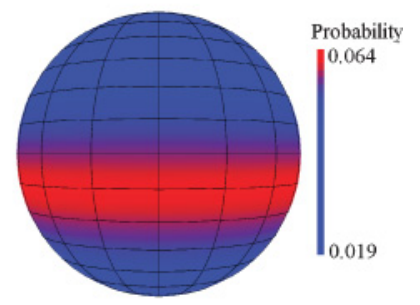

(d)
FIG. 3. (Color) Quantum probability of finding a second electron anywhere on the sphere of radius $N / Z$ a.u. when the first electron is at the north pole for the ground states of various atoms and ions. (a) High-charge ion, beryllium sequence; (c) neutral beryllium; (b) high-charge ion, nitrogen sequence; (d) neutral nitrogen. Plots (a) and (b) show exact pair densities $\rho_{2}(x, y)=\langle\Psi| \delta\left(x-x_{1}\right) \delta(y-$ $\left.x_{2}\right)|\Psi\rangle, \Psi$ from Table I, $x=(0,0, N / Z),|y|=N / Z$; (c) and (d) are based on numerical wave functions [19]. In (a), neglecting the corrections in Table I and Fig. 1 to the standard $1 s^{2} 2 s^{2}$ configuration would lead to the unphysical result of a constant probability.

Schrödinger ground state (Fig. 1) coincides with the hydrogen orbital configuration predicted from (a), (b), and (c). For the remaining three elements $\mathrm{Be}, \mathrm{B}$, and $\mathrm{C}$, the large-ion ground state contains the hydrogen orbital configuration as a dominant part, but a 10 to $20 \%$ admixture of a particular "higher subshell" configuration is also present, in which the $2 s^{2}$ electron pair has migrated to a $2 p$ orbital, see Fig. 2. This shows that rule (b) is not obeyed in a strict sense but only probabilistically. Numerical $a b$ initio computations confirm that this effect persists as $Z$ is decreased to neutrality [19].

These higher subshell contributions turn out to significantly affect the typical relative position of electron pairs, which, as we argue below, is a significant indicator for preferred bond angles and hence chemical behavior.

Figure 3 demonstrates this point for the beryllium sequence. For the quantum state in Fig. 1, and the first electron fixed, without loss of generality, at the north pole on a sphere around the nucleus, the preferred position of the second electron (red) is seen to be at the south pole, but all positions would be equally likely when the higher subshell contributions are ignored. It is interesting to compare with the nitrogen sequence, where the preferred position of the second electron (red) is at a nonlinear angle. This different behavior of Be and $\mathrm{N}$ correlates in a tantalizing way with the experimental fact that the $\mathrm{BeH}_{2}$ molecule is straight, but $\mathrm{NH}_{2}$ is bent. The connection becomes clear when one interprets the shape of the trimer as a rough measurement of the relative position of the two bonding electrons contributed by the central atom. 
TABLE I. An orthonormal basis of ground states of the Schrödinger equations (1) and (2) in the large $Z$ limit in standard notation (see Appendix). The symmetry agrees with experiment for each element of each sequence, including neutral atoms.

\begin{tabular}{|c|c|c|c|}
\hline Isoel. seq. & Sym. & Exact ground state in large $Z$ limit & Dim. \\
\hline $\mathrm{H}$ & ${ }^{2} S$ & $|1 s\rangle,|\overline{1 s}\rangle$ & 2 \\
\hline $\mathrm{He}$ & ${ }^{1} S$ & $\left|1 s^{2}\right\rangle$ & 1 \\
\hline $\mathrm{Li}$ & ${ }^{2} S$ & $\left|1 s^{2} 2 s\right\rangle,\left|1 s^{2} \overline{2 s}\right\rangle$ & 2 \\
\hline $\mathrm{Be}$ & ${ }^{1} S$ & $\begin{array}{l}\frac{1}{\sqrt{1+c^{2}}}\left(\left|1 s^{2} 2 s^{2}\right\rangle+c \frac{1}{\sqrt{3}}\left(\left|1 s^{2} 2 p_{1}^{2}\right\rangle+\left|1 s^{2} 2 p_{2}^{2}\right\rangle+\left|1 s^{2} 2 p_{3}^{2}\right\rangle\right)\right) \\
c=-\frac{\sqrt{3}}{59049}(2 \sqrt{1509308377}-69821)=-0.2310995 \ldots\end{array}$ & 1 \\
\hline $\mathrm{B}$ & ${ }^{2} P^{o}$ & $\begin{array}{c}\frac{1}{\sqrt{1+c^{2}}}\left(\left|1 s^{2} 2 s^{2} 2 p_{i}\right\rangle+c \frac{1}{\sqrt{2}}\left(\left|1 s^{2} 2 p_{i} 2 p_{j}^{2}\right\rangle+\left|1 s^{2} 2 p_{i} 2 p_{k}^{2}\right\rangle\right)\right) \\
\frac{1}{\sqrt{1+c^{2}}}\left(\left|1 s^{2} 2 s^{2} \overline{2 p_{i}}\right\rangle+c \frac{1}{\sqrt{2}}\left(\left|1 s^{2} \overline{2 p_{i}} 2 p_{j}^{2}\right\rangle+\left|1 s^{2} \overline{2 p_{i}} 2 p_{k}^{2}\right\rangle\right)\right) \\
(i, j, k)=(3,1,2),(1,2,3),(2,3,1) \\
c=-\frac{\sqrt{2}}{393660}(\sqrt{733174301809}-809747)=-0.1670823 \ldots\end{array}$ & 6 \\
\hline $\mathrm{C}$ & ${ }^{3} P$ & $\begin{array}{c}\frac{1}{\sqrt{1+c^{2}}}\left(\left|1 s^{2} 2 s^{2} 2 p_{i} 2 p_{j}\right\rangle+c\left|1 s^{2} 2 p_{k}^{2} 2 p_{i} 2 p_{j}\right\rangle\right) \\
\frac{1}{\sqrt{1+c^{2}}}\left(\frac{1}{\sqrt{2}}\left(\left|1 s^{2} 2 s^{2} 2 p_{i} \overline{2 p_{j}}\right\rangle+\left|1 s^{2} 2 s^{2} \overline{2 p_{i}} 2 p_{j}\right\rangle\right)+c \frac{1}{\sqrt{2}}\left(\left|1 s^{2} 2 p_{k}^{2} 2 p_{i} \overline{2 p_{j}}\right\rangle+\left|1 s^{2} 2 p_{k}^{2} \overline{2 p_{i}} 2 p_{j}\right\rangle\right)\right) \\
\frac{1}{\sqrt{1+c^{2}}}\left(\left|1 s^{2} 2 s^{2} \overline{2 p_{i} 2 p_{j}}\right\rangle+c\left|1 s^{2} 2 p_{k}^{2} \overline{2 p_{i} 2 p_{j}}\right\rangle\right) \\
(i, j, k)=(3,1,2),(1,2,3),(2,3,1) \\
c=-\frac{1}{98415}(\sqrt{221876564389}-460642)=-0.1056317 \ldots\end{array}$ & 9 \\
\hline $\mathrm{N}$ & ${ }^{4} S^{o}$ & $\begin{array}{c}\left|1 s^{2} 2 s^{2} 2 p_{1} 2 p_{2} 2 p_{3}\right\rangle \\
\frac{1}{\sqrt{3}}\left(\left|1 s^{2} 2 s^{2} \overline{2 p_{3}} 2 p_{1} 2 p_{2}\right\rangle+\left|1 s^{2} 2 s^{2} 2 p_{3} \overline{2 p_{1}} 2 p_{2}\right\rangle+\left|1 s^{2} 2 s^{2} 2 p_{3} 2 p_{1} \overline{2 p_{2}}\right\rangle\right) \\
\frac{1}{\sqrt{3}}\left(\left|1 s^{2} 2 s^{2} \overline{2 p_{3} 2 p_{1}} 2 p_{2}\right\rangle+\left|1 s^{2} 2 s^{2} \overline{2 p_{3}} 2 p_{1} \overline{2 p_{2}}\right\rangle+\left|1 s^{2} 2 s^{2} 3 \overline{2 p_{1} 2 p_{2}}\right\rangle\right) \\
\left|1 s^{2} 2 s^{2} \overline{2 p_{1} 2 p_{2} 2 p_{3}}\right\rangle\end{array}$ & 4 \\
\hline $\mathrm{O}$ & ${ }^{3} P$ & $\begin{array}{c}\left|1 s^{2} 2 s^{2} 2 p_{i}^{2} 2 p_{j} 2 p_{k}\right\rangle \\
\frac{1}{\sqrt{2}}\left(\left|1 s^{2} 2 s^{2} 2 p_{i}^{2} 2 p_{j} \overline{2 p_{k}}\right\rangle+\left|1 s^{2} 2 s^{2} 2 p_{i}^{2} \overline{2 p_{j}} 2 p_{k}\right\rangle\right) \\
\left|1 s^{2} 2 s^{2} 2 p_{i}^{2} \overline{2 p_{j} 2 p_{k}}\right\rangle \\
(i, j, k)=(3,1,2),(1,2,3),(2,3,1)\end{array}$ & 9 \\
\hline $\mathrm{F}$ & ${ }^{2} P^{o}$ & $\begin{array}{c}\left|1 s^{2} 2 s^{2} 2 p_{i}^{2} 2 p_{j}^{2} 2 p_{k}\right\rangle \\
\left|1 s^{2} 2 \overline{2} 2 p_{i}^{2} 2 p_{j}^{2} \overline{2 p_{k}}\right\rangle \\
(i, j, k)=(3,1,2),(1,2,3),(2,3,1)\end{array}$ & 6 \\
\hline $\mathrm{Ne}$ & ${ }^{1} S$ & $\left|1 s^{2} 2 s^{2} 2 p_{1}^{2} 2 p_{2}^{2} 2 p_{3}^{2}\right\rangle$ & 1 \\
\hline
\end{tabular}

Our exact quantum states also allow theoretical insight into the failure of Hund's rules for certain excited states. Experimentally, the lowest $1 s^{2} 2 s 2 p^{3}{ }^{3} S^{o}$ and ${ }^{1} D^{o}$ levels of the carbon sequence cross between $Z=20$ and $Z=$ 19 , whereas Hund's rules would order them universally as ${ }^{3} S^{o}<{ }^{1} D^{o}$. However, the energy difference as read off from Table III (by writing each energy as $\langle\Psi|H| \Psi\rangle$ and using Slater's rules [15]) consists of a $2 s-2 p$ positive exchange term and a $2 p-2 p$ negative exchange term, $E_{3} S^{o}-E_{1} D^{o}=$ $\left(2 s 2 p_{1} \mid 2 p_{1} 2 s\right)-3\left(2 p_{1} 2 p_{2} \mid 2 p_{2} 2 p_{1}\right)$, and so indeed could have either sign, depending on the orbitals. This interesting effect is missed when these states are modelled by the aufbau principle Slater determinants $\left|1 s^{2} 2 s 2 p_{3} \overline{2 p_{1} 2 p_{2}}\right\rangle$ (singlet) and $\left|1 s^{2} 2 s 2 p_{3} 2 p_{1} \overline{2 p_{2}}\right\rangle$ (triplet). The energy difference is then $E_{3} S^{o}-E_{1} D^{o}=-\left(2 s 2 p_{1} \mid 2 p_{1} 2 s\right)<0$, which wrongly predicts a universal ordering.

\section{ENERGY LEVELS}

The energy levels $E=E_{j}(N, Z)$ of the atom/ion with $N$ electrons and nuclear charge $Z$ have the the following asymptotic expansion for large $Z[3,5-7,14]$

$$
E_{j}(N, Z)=a^{(0)}(N) Z^{2}+a_{j}^{(1)}(N) Z+O(1) .
$$

Here $a^{(0)}(N) Z^{2}$ is a contribution purely from kinetic energy and electron-nucleus attraction, whilst the next order term $a_{j}^{(1)}(N) Z$ stems from electron-electron repulsion. The coefficient $a^{(0)}$ is a sum of hydrogen atom eigenvalues, $a^{(0)}(N)=\sum_{i=1}^{N}-1 / n_{i}^{2}$; for the ground state one has $a_{\mathrm{GS}}^{(0)}(N)=$ $-1-(N-2) / 8$ for $N=2, \ldots, 10$. Much less trivially, we have succeeded in also determining $a_{j}^{(1)}$ in closed form, for all low-lying energy levels of the first 10 atoms (see Table III and the Appendix). We note that the $O(1)$ term in (3) can be 
TABLE II. The 12 lowest asymptotic eigenstates of (1) and (2) for the carbon sequence $(N=6)$, along with their term values and energies, ordered from top to bottom with increasing energy. The theoretical large- $Z$ ordering agrees with experiment for all $Z \geqslant 20$, and differs by a single crossing between the 7th and 8th levels when $Z \leqslant 19$ [see Fig. 4(d)]. Only the states with $L_{3}=0$ and $S_{3}=S$ are shown. The remaining states, and those for the remaining $N=3, \ldots, 10$, are listed in Ref. [14].

\begin{tabular}{|c|c|c|}
\hline Term & $\Psi$ & $E(Z)$ \\
\hline${ }^{3} P$ & $\begin{array}{c}\frac{1}{\sqrt{1+c^{2}}}\left(\left|1 s \overline{1 s} 2 s \overline{2 s} 2 p_{1} 2 p_{2}\right\rangle+c\left|1 s \overline{1 s} 2 p_{3} \overline{2 p_{3}} 2 p_{1} 2 p_{2}\right\rangle\right) \\
c=\frac{460642}{98415}-\frac{\sqrt{221876564389}}{98415}\end{array}$ & $-\frac{3}{2} Z^{2}+\left(\frac{3806107}{1119744}-\frac{\sqrt{221876564389}}{3359232}\right) Z$ \\
\hline${ }^{1} D$ & $\begin{array}{c}\frac{1}{\sqrt{1+c^{2}}}\left(\frac{1}{\sqrt{6}}\left(2\left|1 s \overline{1 s} 2 s \overline{2 s} 2 p_{3} \overline{2 p_{3}}\right\rangle-\left|1 s \overline{1 s} 2 s \overline{2 s} 2 p_{1} \overline{2 p_{1}}\right\rangle-\left|1 s \overline{1 s} 2 s \overline{2 s} 2 p_{2} \overline{2 p_{2}}\right\rangle\right)\right. \\
\left.+c \frac{1}{\sqrt{6}}\left(2\left|1 s \overline{1 s} 2 p_{1} \overline{2 p_{1}} 2 p_{2} \overline{2 p_{2}}\right\rangle-\left|1 s \overline{1 s} 2 p_{3} \overline{2 p_{3}} 2 p_{1} \overline{2 p_{1}}\right\rangle-\left|1 s \overline{1 s} 2 p_{3} \overline{2 p_{3}} 2 p_{2} \overline{2 p_{2}}\right\rangle\right)\right) \\
c=-\frac{460642}{98415}+\frac{\sqrt{221876564389}}{98415}\end{array}$ & $-\frac{3}{2} Z^{2}+\left(\frac{19148633}{5598720}-\frac{\sqrt{221876564389}}{3359232}\right) Z$ \\
\hline${ }^{1} S$ & $\begin{array}{c}\frac{1}{\sqrt{1+c^{2}}}\left(\frac{1}{\sqrt{3}}\left(\left|1 s \overline{1 s} 2 s \overline{2 s} 2 p_{3} \overline{2 p_{3}}\right\rangle+\left|1 s \overline{1 s} 2 s \overline{2 s} 2 p_{1} \overline{2 p_{1}}\right\rangle+\left|1 s \overline{1 s} 2 s \overline{2 s} 2 p_{2} \overline{2 p_{2}}\right\rangle\right)\right. \\
\left.+c\left(\frac{1}{\sqrt{3}}\left(\left|1 s \overline{1 s} 2 p_{3} \overline{2 p_{3}} 2 p_{1} \overline{2 p_{1}}\right\rangle+\left|1 s \overline{1 s} 2 p_{3} \overline{2 p_{3}} 2 p_{2} \overline{2 p_{2}}\right\rangle+\left|1 s \overline{1 s} 2 p_{1} \overline{2 p_{1}} 2 p_{2} \overline{2 p_{2}}\right\rangle\right)\right)\right) \\
c=\frac{230321}{98415}-\frac{\sqrt{62733275266}}{98415}\end{array}$ & $-\frac{3}{2} Z^{2}+\left(\frac{966289}{279936}-\frac{\sqrt{62733275266}}{1679616}\right) Z$ \\
\hline${ }^{5} S^{o}$ & $\left|1 s \overline{1 s} 2 s 2 p_{3} 2 p_{1} 2 p_{2}\right\rangle$ & $-\frac{3}{2} Z^{2}+\frac{464555}{139968} Z$ \\
\hline${ }^{3} D^{o}$ & $\frac{1}{\sqrt{6}}\left(2\left|1 s \overline{1 s} 2 s \overline{2 p_{3}} 2 p_{1} 2 p_{2}\right\rangle-\left|1 s \overline{1 s} 2 s 2 p_{3} 2 p_{1} \overline{2 p_{2}}\right\rangle-\left|1 s \overline{1 s} 2 s 2 p_{3} \overline{2 p_{1}} 2 p_{2}\right\rangle\right)$ & $-\frac{3}{2} Z^{2}+\frac{4730843}{1399680} Z$ \\
\hline${ }^{3} P^{o}$ & $\frac{1}{\sqrt{2}}\left(\left|1 s \overline{1 s} 2 s 2 p_{3} 2 p_{1} \overline{2 p_{1}}\right\rangle+\left|1 s \overline{1 s} 2 s 2 p_{3} 2 p_{2} \overline{2 p_{2}}\right\rangle\right)$ & $-\frac{3}{2} Z^{2}+\frac{1904147}{559872} Z$ \\
\hline${ }^{3} S^{o}$ & $\begin{array}{l}\frac{1}{\sqrt{12}}\left(3\left|1 s \overline{1 s 2 s} 2 p_{3} 2 p_{2} 2 p_{2}\right\rangle-\left|1 s \overline{1 s} 2 s \overline{2 p_{3}} 2 p_{1} 2 p_{2}\right\rangle\right. \\
\left.-\left|1 s \overline{1 s} 2 s 2 p_{3} \overline{2 p_{1}} 2 p_{2}\right\rangle-\left|1 s \overline{1 s} 2 s 2 p_{3} 2 p_{1} \overline{2 p_{2}}\right\rangle\right)\end{array}$ & $-\frac{3}{2} Z^{2}+\frac{961915}{279936} Z$ \\
\hline${ }^{1} D^{o}$ & $\begin{array}{l}\frac{1}{\sqrt{12}}\left(2\left|1 s \overline{1 s} 2 s 2 p_{3} \overline{2 p_{1} 2 p_{2}}\right\rangle-\left|1 s \overline{1 s} 2 s \overline{2 p_{3}} 2 p_{1} \overline{2 p_{2}}\right\rangle-\left|1 s \overline{1 s} 2 s \overline{2 p_{3} 2 p_{1}} 2 p_{2}\right\rangle\right. \\
\left.+2\left|1 s \overline{1 s 2 s 2 p_{3}} 2 p_{1} 2 p_{2}\right\rangle-\left|1 s \overline{1 s 2 s} 2 p_{3} 2 p_{1} \overline{2 p_{2}}\right\rangle-\left|1 s \overline{1 s 2 s} 2 p_{3} \overline{2 p_{1}} 2 p_{2}\right\rangle\right)\end{array}$ & $-\frac{3}{2} Z^{2}+\frac{9625711}{2799360} Z$ \\
\hline${ }^{1} P^{o}$ & $\begin{array}{l}\frac{1}{2}\left(\left|1 s \overline{1 s} 2 s \overline{2 p_{3}} 2 p_{1} \overline{2 p_{1}}\right\rangle-\left|1 s \overline{1 s 2 s} 2 p_{3} 2 p_{1} \overline{2 p_{1}}\right\rangle\right. \\
\left.+\left|1 s \overline{1 s} 2 s \overline{2 p_{3}} 2 p_{2} \overline{2 p_{2}}\right\rangle-\left|1 s \overline{1 s 2 s} 2 p_{3} 2 p_{2} \overline{2 p_{2}}\right\rangle\right)\end{array}$ & $-\frac{3}{2} Z^{2}+\frac{242119}{69984} Z$ \\
\hline${ }^{3} P$ & Same as ${ }^{3} P$ above, $c=\frac{460642}{98415}+\frac{\sqrt{221876564389}}{98415}$ & $-\frac{3}{2} Z^{2}+\left(\frac{3806107}{1119744}+\frac{\sqrt{221876564389}}{3359232}\right) Z$ \\
\hline${ }^{1} D$ & Same as ${ }^{1} D$ above, $c=-\frac{460642}{98415}-\frac{\sqrt{221876564389}}{98415}$ & $-\frac{3}{2} Z^{2}+\left(\frac{19148633}{5598720}+\frac{\sqrt{221876564389}}{3359232}\right) Z$ \\
\hline${ }^{1} S$ & Same as ${ }^{1} S$ above, $c=\frac{230321}{98415}+\frac{\sqrt{62733275266}}{98415}$ & $-\frac{3}{2} Z^{2}+\left(\frac{966289}{279936}+\frac{\sqrt{62733275266}}{1679616}\right) Z$ \\
\hline
\end{tabular}

expanded further into

$$
a_{j}^{(2)}(N)+a_{j}^{(3)}(N) \frac{1}{Z}+\cdots ;
$$

but already the next order coefficients $a_{j}^{2}(N)$ are not known exactly even for $N=2$ (for numerical values see, e.g., Ref. [26]). Hence our closed-form energies $a^{(0)}(N) Z^{2}+a_{j}^{(1)}(N) Z$, unlike

TABLE III. Missing experimental energy levels predicted from Fig. 4, along with their symmetry and the dominant configuration.

\begin{tabular}{crcccc}
\hline \hline$N$ & $Z$ & Atom or ion & Domin. conf. & Sym. & $E_{i}-E_{1}$ (a.u.) \\
\hline 6 & 15 & P X & $1 s^{2} 2 p^{4}$ & ${ }^{1} S$ & 4.0831 \\
6 & 18 & Ar XIII & $1 s^{2} 2 p^{4}$ & ${ }^{1} S$ & 5.2334 \\
6 & 18 & Ar XIII & $1 s^{2} 2 s 2 p^{3}$ & ${ }^{5} S^{o}$ & 0.9715 \\
6 & 6 & $\mathrm{C}$ & $1 s^{2} 2 s^{2} 2 p^{2}$ & ${ }^{3} P$ & $0.68 \pm 0.004$ \\
6 & 6 & $\mathrm{C}$ & $1 s^{2} 2 s^{2} 2 p^{2}$ & ${ }^{1} D$ & $0.76 \pm 0.004$ \\
6 & 6 & $\mathrm{C}$ & $1 s^{2} 2 s^{2} 2 p^{2}$ & ${ }^{1} S$ & $0.85 \pm 0.004$ \\
7 & 18 & $\mathrm{Ar} \mathrm{XII}$ & $1 s^{2} 2 s 2 p^{4}$ & ${ }^{2} P^{o}$ & 5.3458 \\
8 & 17 & $\mathrm{Cl} \mathrm{X}$ & $1 s^{2} 2 s 2 p^{5}$ & ${ }^{1} P^{o}$ & 3.0407 \\
\hline \hline
\end{tabular}

our closed-form wave functions, do not have an asymptotically vanishing absolute error but only an asymptotically vanishing relative error.

To compare the asymptotic result (3) to experimental energy levels, we argue that it is useful not to make a comparison of bare values, or bare gaps, but to first renormalize both the energy gaps and the nuclear charge $Z$ so as to make them constant at $Z=\infty$. We see from (3) that this is achieved by the following prescription:

Do not plot $E_{j}(Z)-E_{1}(Z)$ against $Z$,

$$
\text { but } \frac{E_{j}(Z)-E_{1}(Z)}{Z^{2}} \text { against } \frac{1}{Z} \text {. }
$$

This scaling, which is a natural application of "renormalization group thinking", also reveals a wealth of hidden structure in the experimental spectra.

Theoretical predictions from (3) and Table II are as follows:

(i) The energy levels should form smooth curves as a function of $1 / Z$. This is a consequence [27] of the smooth dependence of the Hamiltonian (2) on $Z$ and smoothness of the rescaling (5). 


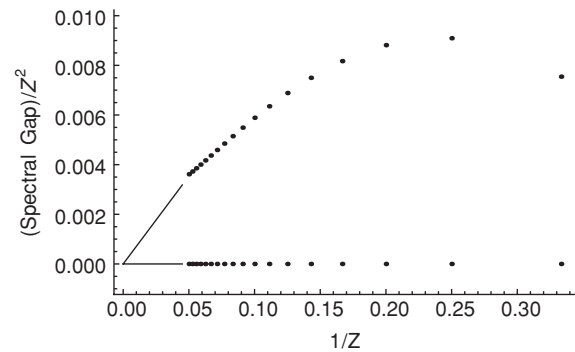

(a)

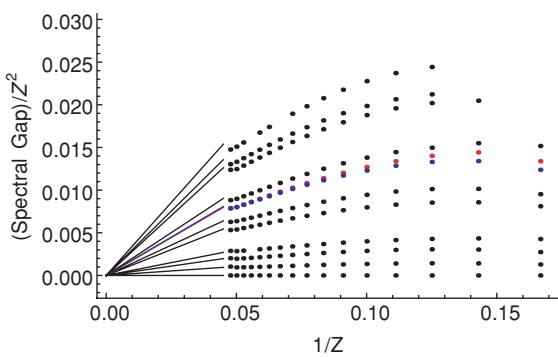

(d)

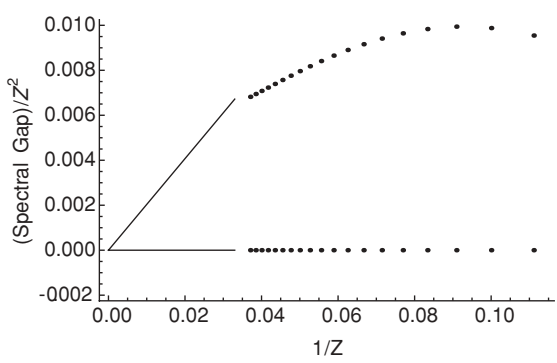

(g)

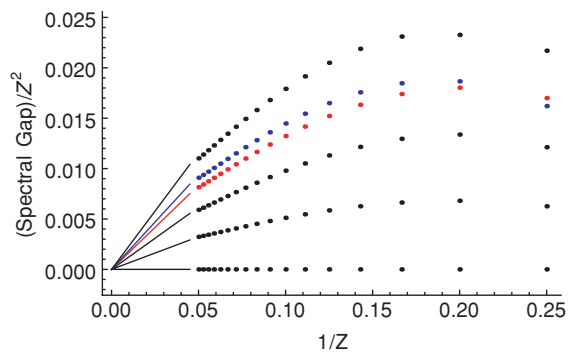

(b)

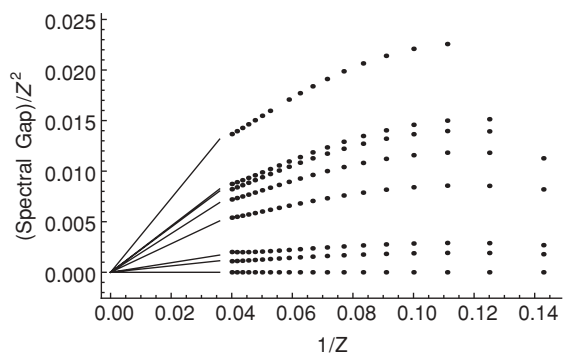

(e)

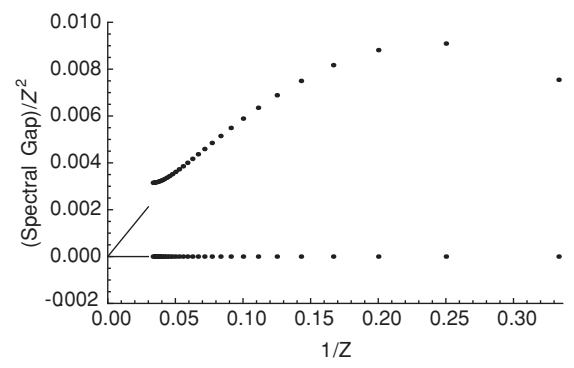

(h)

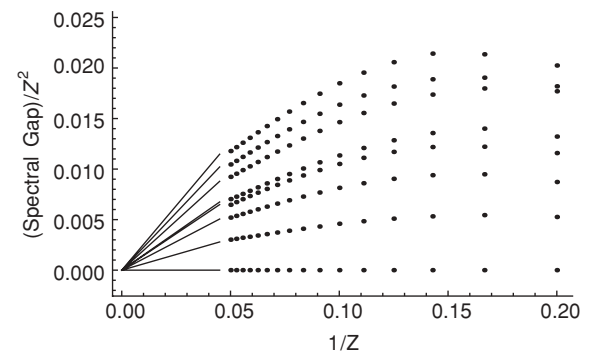

(c)

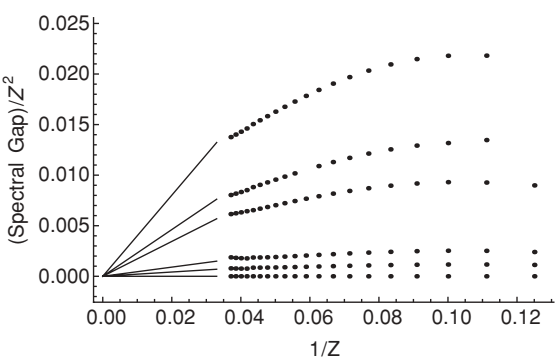

(f)

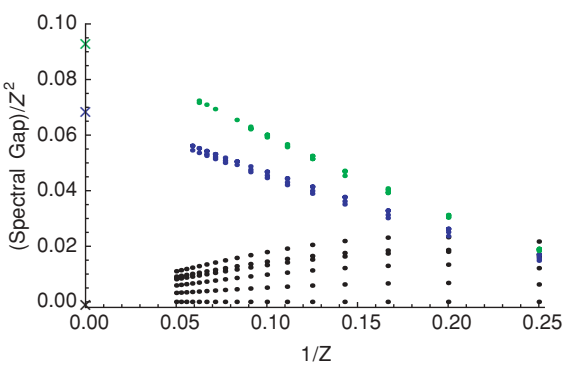

(i)

FIG. 4. (Color) (a)-(g): Energy levels of the isoelectronic sequences with three to nine electrons. (Lines) Asymptotic Schrödinger levels (this article); (points) experimental data [20] (averaged, by multiplicity, over J); only levels associated to $1 s^{2} 2 s^{i} 2 p^{N-2-i}$ configurations are shown. To reveal the close similarity of spectra across each isoelectronic sequence, the natural but previously unused scaling (5) is essential. Note that only two level crossings are present (in the beryllium and carbon sequences, colored red and blue). (h) Relativistic effects in the lithium sequence for $Z \gtrsim 20$. (i) Higher principal quantum number levels in the beryllium sequence and their theoretical limits $(5 / 72$ for $1 s^{2} 2 s^{2} 3 \ell$ and $3 / 22$ for $1 s^{2} 2 s^{2} 4 \ell, \ell=s, p$; blue and green points and crosses, respectively).

(ii) The number of curves converging to zero as $1 / Z \rightarrow 0$ should correspond exactly to the number $n(N)$ of different $a_{j}^{(1)}$ possible in formula (3) when $a^{(0)}$ is given by its lowest value $a_{\mathrm{GS}}^{(0)}$. By our results, $n(N)$ equals the number of eigenvalues of the reduced Hamiltonian (A1) (for $2 \leqslant N \leqslant 10$, $1,2,6,8,12,8,6,2,1)$.

(iii) The exact theoretical tangents at $1 / Z=0$ to the energy gap curves are given by $t_{j}\left(\frac{1}{Z}\right)=\left(a_{j}^{(1)}(N)-a_{1}^{(1)}\right) / Z$, with the $a_{j}^{(1)}$ as in Table III.

(iv) Energy curves whose dominant configurations contain higher principal quantum number orbitals should converge to values greater than zero as $1 / Z \rightarrow 0$, more explicitly to $a^{(0)}(N)-a_{\mathrm{GS}}^{(0)}(N)$. These predictions are all beautifully confirmed by the experimental data, see Fig. 4 .

Such plots [see Fig. 3 (h)] also clearly demonstrate, for $Z \gtrsim 20$, relativistic deviations from (1) and (2) (theoretically, energy corrections scale like $\alpha^{2} Z^{4}$ as Z gets large, where $\alpha \approx$ $1 / 137$ is the fine structure constant).
Finally we discuss the error made by neglecting the higher-order asymptotic energy corrections in (4). As Fig. 4 and Table III demonstrate, further terms are not needed to resolve the number of levels emanating from the noninteracting ground state, along with their term values and orderings. The size of the next order $O(1)$ term is known numerically in some cases, such as the asymptotically lowest $\left({ }^{1} S\right.$, dominant configuration $\left.1 s^{2} 2 s^{2}\right)$, third $\left({ }^{1} P^{o}\right.$, domin. conf. $\left.1 s^{2} 2 s 2 p\right)$ and sixth $\left({ }^{1} S\right.$, domin. conf. $\left.1 s^{2} 2 p^{2}\right)$ levels of the Be sequence in Fig. 4(b) [28]:

\begin{tabular}{lcccc}
\hline \hline Gap & ${ }^{1} P^{o}-{ }^{1} S$ & ${ }^{1} S-{ }^{1} S$ & ${ }^{1} P^{o}-{ }^{1} S$ & ${ }^{1} S-{ }^{1} S$ \\
\hline$Z$ & 4 & 4 & 20 & \multicolumn{1}{c}{20} \\
$O\left(Z^{2}\right)+O(Z)$ & 0.4939 & 0.9252 & 2.4693 & 4.6260 \\
contrib. [14] & & & & \\
$O(1)$ contrib. [28] & -0.2103 & -0.3638 & -0.2103 & -0.3638 \\
Experimental & 0.1939 & 0.3471 & 2.3630 & 4.4068 \\
value [20] & & & & \\
\hline
\end{tabular}


Thus the $O(1)$ term in (4) is important for neutral atoms, as expected, while for highly charged ions with $Z \approx 20$, the error with or without this term is of the same magnitude (e.g., -0.104 versus +0.106 for the ${ }^{1} P^{o}-{ }^{1} S$ Be sequence gap). Hence in order to significantly improve our theoretical energies in this regime, relativistic effects would need to be taken into account as well.

\section{TERM AND CONFIGURATION ASSIGNMENT FOR NEUTRAL ATOMS}

We now turn to neutral atoms and the important issue of assigning term values (total spin, angular momentum, and parity quantum numbers $L, S$, and $p$, encoded as ${ }^{2 S+1} X^{v}$, where $v$ indicates parity and $X=S, P, D, \ldots$ means $L=$ $0,1,2, \ldots)$ to observed energy levels.

We propose an alternative to standard assignment methods [22], which carefully exploits both experimental and theoretical insights on the large $Z$ limit.

(1) Plot the experimental excitation energies along an isoelectronic sequence, under the scaling (5), as in Fig. 4.

(2) Near $1 / Z=0$ our exact results (see Table II) deliver closed-form wave functions for each level and hence unambiguous term values and configurations.

(3) The term values remain constant along each energy level curve, since $L, S$, and $p$ are quantized and hence their continuous dependence on the parameter $Z$ forbids them to change.

(4) Ambiguities arise only when continuing term assignments through level crossings. These can be resolved by a simple and theoretically justified curvature minimization algorithm, described below.

We have applied this method to the 45 energy levels emanating from the ground states of the atoms $\mathrm{Li}$ to $\mathrm{Ne}$ at $1 / Z=0$, obtaining the experimentally established term value in each case. For the corresponding ions, our method captured correctly the crossings in the carbon sequence between $Z=19$ and 20, the beryllium sequence between $Z=4$ and 5 , and the noncrossing despite visual ambiguity in the boron sequence between $Z=5$ and 6 .

Our method also assigns a definite "configuration" (e.g., $1 s^{2} 2 s^{2} 2 p^{3}$ ) to an atomic quantum state: namely the configuration associated to the corresponding energy level curve in the limit $1 / Z \rightarrow 0$. This procedure can be thought of as an alternative, less empirical definition of the notion of configuration, not as an approximate property of the state (obtained as a best fit of experimental data to model wave functions) but as an exact property of the deformed state which emerges when one makes the nuclear charge large.

Our curvature minimization algorithm (4) continues term assignment iteratively from $Z$ to $Z-1$ by considering an arbitrary possible pairing of levels at $Z$ with those at $Z-1$, connecting each pair by a cubic spline $\tilde{E}_{i}(s)(Z-1 \leqslant s \leqslant Z)$ and minimizing the resulting total level curvature $C(Z-1, Z)=$ $\int_{Z-1}^{Z} \sum_{i=1}^{N}\left[\tilde{E}_{i}^{\prime \prime}(s)\right]^{2} d s$ over all matchings. This algorithm has its theoretical basis in the following simple mathematical result: with the $\tilde{E}_{i}$ replaced by the exact Schrödinger levels and assuming any crossings are transverse, $C$ is finite only for the correct labeling, and infinite otherwise, due to kink singularities at crossings. In practice, we found it sufficient to interpolate by cubic splines.

This method also allows the prediction of missing experimental values by taking the value given by the cubic spline at the appropriate value of $1 / Z$; see Fig. 4. Due to the lack of constraints for the cubic spline fitting, "end" values at $Z=N$ are harder to predict, as indicated by the error bar for the carbon levels.

\section{CORRECTION OF EXPERIMENTAL TERM ASSIGNMENTS}

Our results strongly suggest that two levels of the 5electron isoelectronic sequence are incorrectly assigned in the NIST database [20]. The levels in question are assigned to the $1 s^{2} 2 s 2 p^{2}$ configuration, with term values ${ }^{2} S J=1 / 2$ and ${ }^{2} P \quad J=1 / 2,3 / 2$. For $Z \leqslant 22$, the two $J=1 / 2$ terms (experimentally indistinguishable through multiplicity) are assigned with ${ }^{2} S<{ }^{2} P$, whereas for $Z \geqslant 23$ (the ions V XIX, Cr XX, Mn XXI, Fe XXII, Co XXIII, Ni XXIV) the order is reversed. We suggest here that this reversal is incorrect; the modified data gives much more convincingly smooth curves, see Fig. 5. By contrast, the analogous ${ }^{2} S$ and ${ }^{2} P$ levels of
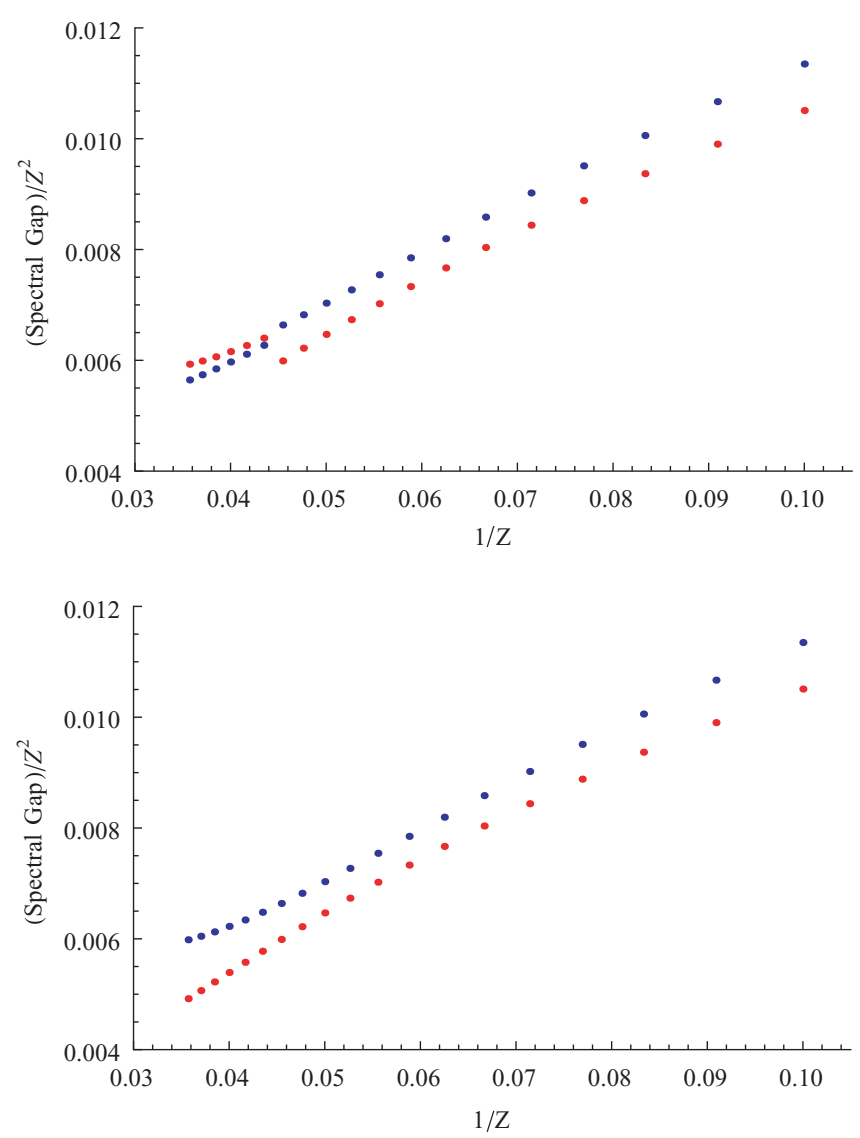

FIG. 5. (Color) Experimental energies of five-electron ions as assigned by NIST, averaged over $J$ (top), and with the ${ }^{2} S$ and ${ }^{2} P$ $J=1 / 2$ levels transposed for $Z \geqslant 23$ before averaging (bottom). The NIST assignment appears to be at odds with the principle that the levels should lie on smooth curves. 
seven-electron ions with configuration $1 s^{2} 2 s 2 p^{4}$ appear to be correctly assigned.

\section{CONCLUSIONS}

For highly charged ions, hydrogen orbital configurations do not just arise as semiempirical approximations but as exact quantum states, which emerge directly from the Schrödinger equation along with definite term values, energetic orderings, and same-shell-higher-subshell corrections. These states, when combined with the systematic study of properties of interest across isoelectronic sequences, provide a widely applicable route to accessing atomic structure and complex spectra. Another use of our results, as rare benchmark data for the design of computational methods, will be explored elsewhere [19].

\section{APPENDIX}

\section{Notation}

In Fig. $1,1 s, 2 s, 2 p_{i}(i=1,2,3)$ are the usual hydrogen orbitals for ions with nuclear charge $Z$ and one electron, $1 s(x)=Z^{3 / 2} e^{-Z|x|} / \sqrt{\pi}, 2 s(x)=Z^{3 / 2}(1-Z|x| / 2) e^{-Z|x| / 2} /$ $\sqrt{8 \pi}, 2 p_{i}(x)=Z^{5 / 2}\left(x \cdot e_{i}\right) e^{-Z|x| / 2} / \sqrt{32 \pi}$. Note that the diameter of the orbitals is smaller by a factor $1 / Z$ as compared to those in neutral hydrogen atoms. Here $x$ is a 3D position coordinate, $e_{1}, e_{2}, e_{3}$ are orthonormal basis vectors of $3 \mathrm{D}$ space, the overbar or its absence gives the spin state (down and up, respectively), a square (as in $1 s^{2}$ ) indicates that both spin states are occupied, and $\left|\varphi_{1} \cdots \varphi_{N}\right\rangle$ stands for the Slater determinant of the orbitals $\varphi_{1}, \ldots, \varphi_{N}$.

\section{Reduction to a finite-dimensional problem}

Use of rescaled position coordinates $\tilde{x}=Z^{-1} x$ removes the factor $Z$ from (2) and creates a small factor, $1 / Z$, in front of electron interaction. Applying degenerate first-order perturbation theory and scaling back to the original variables yields that for large $Z$, the lowest eigenvalues and eigenstates of (1) and (2) have the asymptotic expansion (3), $\Psi=$ $\Psi_{j}(N, Z)=\Phi_{j}^{(0)}(N, Z)+O\left(Z^{-1}\right)$, where the approximate Schrödinger levels $a^{(0)} Z^{2}+a_{j}^{(1)} Z$ and states $\Phi_{j}^{(0)}$ are the exact eigenvalues and eigenstates of the finite-dimensional reduced problem

$$
\text { (a') } P H P \Psi=E \Psi, \quad\left(b^{\prime}\right) \Psi \in V_{0} .
$$

Here $P=\sum_{i}\left|\Psi_{i}\right\rangle\left\langle\Psi_{i}\right|$ is the projector onto the noninteracting ground state $V_{0}$ (lowest eigenspace of (2) with second term deleted). Rule ( $\left.b^{\prime}\right)$ is the same as (a) in the Aufbau principle, while $\left(\mathrm{a}^{\prime}\right)$ replaces the empirical postulates (b) and (c), instead selecting the correct hydrogen orbital configurations from theory.

\section{Determining the eigenvalues and eigenstates of the reduced Hamiltonian $\boldsymbol{P H P}$}

For $2 \leqslant N \leqslant 10$, by hydrogen atom theory plus the theory of noninteracting fermions $V_{0}$ has a basis $\left\{\Psi_{1}, \ldots, \Psi_{d(N)}\right\}$ of Slater determinants with hydrogen orbital configurations $(1 s)^{2}(2 s)^{j}(2 p)^{N-2-j}, j=0,1,2$ and dimension $d=\left(\begin{array}{c}8 \\ N-2\end{array}\right)$ corresponding to the number of possible assignments of the $N-2$ valence electrons to the 8 valence orbitals. $P H P$ is a $d \times d$ matrix with entries $\left\langle\Psi_{i}|H| \Psi_{j}\right\rangle$ (for the carbon sequence, a $70 \times 70$ matrix whose entries are integrals over $\mathbb{R}^{18} \times \mathbb{Z}_{2}^{6}$ ). By conservation of total spin, angular momentum, and parity under (2) [and (A1)], $P H P$ leaves the simultaneous eigenspaces of $\underline{L}^{2}, L_{3}, \underline{S}^{2}, S_{3}$, and parity invariant. Aided by representation theory for the underlying symmetry group $\mathrm{SO}(3) \times \mathbb{Z}_{2} \times \mathrm{SU}(2)$ of (1), (2), (A1) (which corresponds to rotation and inversion of electron positions and rotation of spins), these can be explicitly determined [14] (for previous analysis of some cases, see Ref. [22]). The largest such spaces turn out to be $2 \mathrm{D}$ ! Evaluating the matrix elements of $P H P$ is achieved by successively reducing the domain of integration from $\mathbb{R}^{3 N}$ to $\mathbb{R}^{6}$ to $\mathbb{R}^{3}$ to $\mathbb{R}$ via Slater's rules [15], Fourier analysis, and spherical polar coordinates and evaluating the remaining one-dimensional integrals by residue calculus as implemented in MAPLE.
[1] H. F. Beyer, H.-J. Kluge, and V. P. Shevelko, X-ray Radiation of Highly Charged Ions (Springer, Berlin, 1997).

[2] Fred J. Currell, The Physics of Multiply and Highly Charged Ions, Vols. I \& II (Springer, Berlin, 2003).

[3] E. A. Hylleraas, Z. Phys. 65, 209 (1930).

[4] D. Layzer, Ann. Phys. 8, 271 (1959).

[5] S. Seung and E. B. Wilson, J. Chem. Phys. 47, 5343 (1967).

[6] C. S. Sharma and C. A. Coulson, Proceedings of the Physical Society 80, 81 (1962).

[7] S. Wilson, J. Phys. B: At. Mol. Phys. 17, 505 (1984).

[8] S. Fritzsche, P. Indelicato, and Th. Stohlker, J. Phys. B 38, S707 (2005).

[9] N. Tragin, J.-P. Geindre, C. Chenais-Popovics, J.-C. Gauthier, J.-F. Wyart, and E. Luc-Koenig, Phys. Rev. A 39, 2085 (1989).

[10] M. Maul, A. Schäfer, W. Greiner, and P. Indelicato, Phys. Rev. A 53, 3915 (1996)
[11] N. Bohr, The Theory of Atomic Spectra and Atomic Constitution (Cambridge University Press, New York, 1922).

[12] F. Hund, Zeitschrift für Physik 33, 345 (1925).

[13] J. C. Slater, Quantum Theory of Atomic Structure (McGraw-Hill, 1960).

[14] G. Friesecke and B. D. Goddard, SIAM J. Math. Anal. 41, 631 (2009).

[15] T. Helgaker, P. Joergensen, and J. Olsen, Molecular Electronic Structure Theory (Wiley, New York, 2000).

[16] B. Bories, D. Maynau, and M.-L. Bonnet, J. Comput. Chem. 28, 632 (2006).

[17] H. Dachsel, R. J. Harrison, and D. A. Dixon, J. Phys. Chem. A 103, 152 (1999).

[18] G. Friesecke, Proc. R. Soc. London A 459, 47 (2003).

[19] G. Friesecke and B. D. Goddard, Multiscale Model. Simul. 7, 1876 (2009). 
[20] Yu. Ralchenko, F.-C. Jou, D. E. Kelleher, A. E. Kramida, A. Musgrove, J. Reader, W. L. Wiese, and K. Olsen, NIST Atomic Spectra Database (version 3.1.2) (National Institute of Standards and Technology, Gaithersburg, MD, 2007).

[21] P. Atkins, Physical Chemistry (Oxford University Press, New York, 2001).

[22] E. U. Condon, Atomic Structure (Cambridge University Press, New York, 1980).

[23] D. Pettifor, Bonding and Structure of Molecules and Solids (Oxford University Press, New York, 1995).
[24] F. Seitz and D. Turnbull, Solid State Physics: Advances in Research and Applications (Academic Press, 1997).

[25] C. Froese Fischer, The Hartree-Fock Method for Atoms. A Numerical Approach (Wiley Interscience, New York, 1977).

[26] J. D. Baker, D. E. Freund, R. N. Hill, and J. D. Morgan III, Phys. Rev. A 41, 1247 (1990).

[27] T. Kato, Perturbation Theory for Linear Operators (Springer, Berlin, 1967).

[28] K. D. Watson and S. V. ONeil, Phys. Rev. A 12, 729 (1975). 